\title{
Critical examination of prewetting and surface melting on caprolactam
}

\author{
Gerard C. L. Wong and Wim H. de Jeu \\ FOM Institute for Atomic and Molecular Physics, Kruislaan 407, 1098SJ Amsterdam, The Netherlands
}

Gertjan Bögels, Erwin P. G. van den Berg, and Piet Bennema

Research Institute for Materials, University of Nijmegen, 6525 ED Nijmegen, The Netherlands

(Received 3 September 1996; revised manuscript received 7 October 1996)

\begin{abstract}
In a previous study, surface melting on the (001) surface of caprolactam $\left(\mathrm{C}_{6} \mathrm{H}_{11} \mathrm{ON}\right)$ was reported to proceed in a series of layering transitions, followed by a prewetting transition. We have reproduced the original results and shown that they can be better explained by the wetting of hydrated caprolactam (with $\mathrm{H}_{2} \mathrm{O}$ molecules at bridging sites between the caprolactam dimers) on caprolactam, and that such hydration is most likely caused by $\mathrm{H}_{2} \mathrm{O}$ microinclusions in the crystals grown from solution. In agreement with this interpretation, we have also shown that the reported effects are absent on “dry" surfaces. [S0163-1829(97)03403-6]
\end{abstract}

Surface melting is a continuous and reversible process by which a positional disordering of the solid crystalline lattice occurs at the solid-vapor interface, at temperatures below the bulk melting temperature $T_{m}$. As the temperature approaches $T_{m}$, the thickness of this disordered region, or "quasiliquid layer," diverges and the solid "melts" inward from the surface. The dominant interactions in the particular physical system determine the dynamics of the solid-liquid and liquid-vapor interfaces, and enforce different functional forms for the divergence of the quasilayer thickness, which have been experimentally observed in various atomic systems. ${ }^{1}$ Long-ranged van der Waals interactions result in a power-law $\left(T_{m}-T\right)^{-1 / 3}$ divergence, while short-ranged interactions (phenomenologically expressed as the spreading parameter $b=\gamma_{\mathrm{sv}}-\left(\gamma_{\mathrm{sl}}+\gamma_{\mathrm{lv}}\right)$, where $\gamma_{\mathrm{sv}}, \gamma_{\mathrm{sl}}$, and $\gamma_{\mathrm{lv}}$ denote the solid-vapor, solid-liquid, and liquid-vapor interface energies) give a logarithmic divergence instead.

Surface melting in anisotropic molecular solids can differ from the atomic systems discussed above, as it may be possible for the orientational order of the component molecules to couple to the translational order. This presents new contexts for the wetting behavior of structured fluids, and may occasion new classes of surface melting. There have been two extant investigations of surface melting in anisotropic van der Waals solids. The first is an ellipsometry study of biphenyl. ${ }^{2}$ Recent experiments, however, have shown that the reported effects are most likely due to extrinsic causes rather than to surface melting. ${ }^{3}$ The second is an x-ray study of the (001) surface of caprolactam $\left(\mathrm{C}_{6} \mathrm{H}_{11} \mathrm{ON}\right)$, which exhibited behavior similar to surface melting. ${ }^{4}$ In the present investigation, we have repeated the original experiment under more controlled conditions, and shown that the observed phenomenon is inconsistent with surface melting. Further, we propose a surface hydration mechanism that can account for all the empirical observations.

Single crystals of caprolactam were grown from solution using a toluene-xylene mixture, as in the original study. ${ }^{5}$ The caprolactam samples used in this study were obtained by cleavage parallel to the (001) surface in a dry $\mathrm{N}_{2}$ environment and then mounted into the inner stage of a two-stage oven. To prevent excessive evaporation from the crystal sur- face, the outer stage of the oven was loaded with freshly powdered dry caprolactam. The temperatures of the ovens were measured with Pt-100 resistors, and calibrated to the bulk $T_{m}$. The sample temperature stability in the inner oven was estimated to be $\sim 50 \mathrm{mK}$, after a typical equilibration time of $1 \mathrm{~h}$.

$\mathrm{X}$-ray specular reflectivity (XSR) experiments probe the average projected electron density $\rho(z)$ along the surface normal direction. ${ }^{6}$ We measured the XSR of the caprolactam (001) surface at different temperatures, by coupling the oven system to a triple-axis spectrometer. The incident $\mathrm{Cu} K \alpha$ beam was monochromatized and focused using a bent graphite crystal, giving a resolution of $\Delta q_{z}=5.7 \times 10^{-3} \AA^{-1}$ along the surface normal direction. The data shown in Fig. 1 have been background subtracted, and the standard geometrical correction for the beam footprint has been applied. Data points for a weak Bragg peak near $Q=0.235 \AA^{-1}$ due to $\lambda / 3$ beam contamination from bremsstrahlung have been omitted. The full width at half maximum of a typical transverse rocking scan across the specular ridge is $\sim 0.045^{\circ}$.

If a layer with a different electron density wets the bulk solid surface, then specular reflections from the top and bottom interfaces of this layer interfere, and thereby modulate the standard Fresnel reflectivity with oscillations. The period of these oscillations, or Kiessig fringes, is determined by the layer thickness, while the amplitude is determined by the electron density difference. Reference 4 reported a series of XSR measurements of the caprolactam (001) surface that had exhibited such intensity oscillations, and therefore indicated the existence of a wetting layer, the thickness of which increased as $T_{m}$ was approached. This behavior of the wetting layer was interpreted within the framework of surface melting. At $\sim 18 \mathrm{~K}$ below $T_{m}$, a weak, slowly varying feature in the reflectivity appeared, which can indicate either a layering transition of the quasi-liquid layer or surface roughening. Since the statistical spread of the data is large, and the original reflectivity measurement only spanned a small range of momentum transfers, the authors acknowledged that both interpretations are possible. A distinctive, discontinuous jump in the wetting layer thickness to $\sim 58 \AA$, however, oc- 
curred at $\sim 13 \mathrm{~K}$ below $T_{m}$, which was unambiguously indicated by two Kiessig fringes. Depending on whether the initial layering transition exists or not, this may be evidence for either a first-order wetting or prewetting transition of the quasiliquid layer. Furthermore, the characteristic thickness at this transition was quite reproducible, and was observed repeatedly during various heating and cooling cycles. At temperatures closer to $T_{m}$, the quasiliquid layer exhibited a continuous increase, although it was not possible to confirm the expected $\left(T_{m}-T\right)^{-1 / 3}$ dependence of the quasi-liquid layer thickness close to $T_{m}$, which is the form required by nonretarded, long-ranged van der Waals interactions.

In contrast to the original experiment, where the surfaces of as-grown crystals were used, we repeated the XSR measurements using caprolactam (001) surfaces of samples that were cleaved and mounted in dry $\mathrm{N}_{2}$. We found no intensity oscillations and therefore no evidence for the existence of a wetting layer or surface melting, up to a temperature of $3.5 \mathrm{~K}$ below $T_{m}$, above which the sample deteriorated rapidly. In order to reconcile these findings with those of Ref. 4, we must consider other explanations for the observed behavior of the wetting layer, in addition to surface melting. Effects related to sample impurity, for example, may be a possible alternative interpretation of the data. However, before such an interpretation can be made, we need to establish (1) the existence of the impurity and the specific forms that it takes, (2) a plausible mechanism for the transport of this impurity to the surface, and (3) a description of a physical process at the surface involving the impurity that can explain the experimental results better than the original proposal of surface melting.

Recent findings indicate that caprolactam crystals grown from solvent solutions, such as the toluene-xylene mixture used in Ref. 4, typically have $0.1-0.2 \%$ of $\mathrm{H}_{2} \mathrm{O}$ contamination in the form of filled crystallographic inclusions, which are $\sim 10 \mu \mathrm{m}$ in diameter. ${ }^{7}$ The mean distance between such inclusions is $\sim 100 \mu \mathrm{m}$, and can be even smaller near the surface compared to the bulk, so that the effective concentration of such inclusions near the surfaces of as-grown samples can be larger than that for cleaved samples. In Ref. 4 , the top layers of the caprolactam sample had been deliberately evaporated before the experiment in order to clean the surface. In addition, more than $50 \mu \mathrm{m}$ of caprolactam can be evaporated during a typical experiment. Given the above, it is conceivable that subsurface inclusions are progressively excavated during an experiment, and eventually lead to high levels of surface contamination by $\mathrm{H}_{2} \mathrm{O}$.

To test this hypothesis, we repeated the experiment with a caprolactam sample cleaved and mounted in dry $\mathrm{N}_{2}$, but this time the sample was kept at $4.0 \mathrm{~K}$ below $T_{m}$ to deliberately evaporate the crystal. Figure 1 shows a sequence of specular reflectivity measurements at different times after temperature equilibration at $T=T_{m}-4.0 \mathrm{~K}$. No oscillations are observed in the reflectivity up to $15 \mathrm{~h}$ after the initial temperature equilibration. However, two Kiessig fringes, which correspond to those reported in the original work, are clearly present at about $45 \mathrm{~h}$ after initial temperature equilibration. Even without the aid of fits, it can be seen that the fringe spacing corresponds to a wetting layer thickness of $\sim 60 \AA$, which is similar to that reported in Ref. 4. The dynamics of this wetting layer cannot possibly be attributed to effects

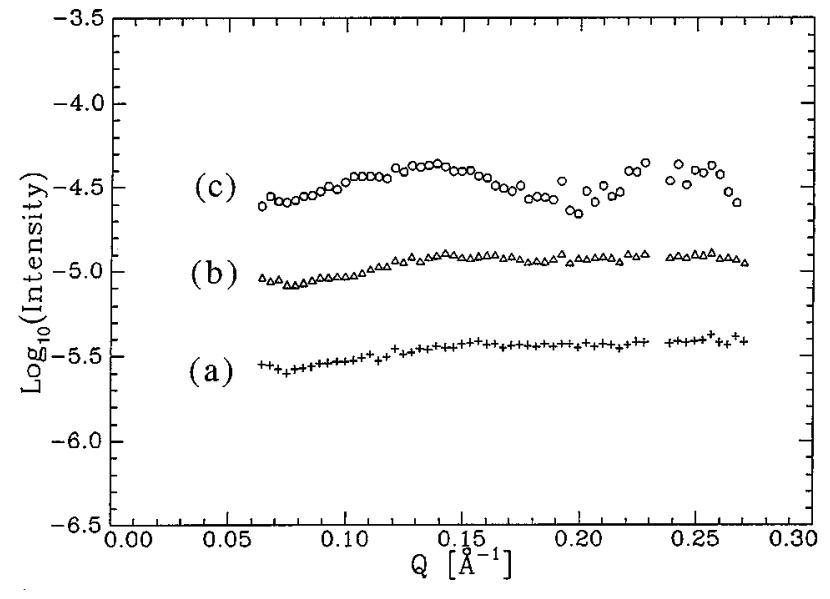

FIG. 1. XSR of caprolactam (001) at $T_{m}-4.0 \mathrm{~K}$ after initial temperature equilibration. (a) After $11 \mathrm{~h}$, (b) after $23 \mathrm{~h}$, (c) after 45 $\mathrm{h}$. The data have been normalized to the asymptotic form for the Fresnel reflectivity: $R(Q) / R_{F}(Q)$ is plotted, where $R_{F}(Q)$ $=\left(Q_{c} / 2 Q\right)^{4}$. Kiessig fringes similar to those reported in Ref. 4 are clearly observed in (c), and indicate the presence of a similar wetting layer $\sim 60 \AA$ thick.

related to temperature equilibration, due to the large observed time scales. Experiments with cleaved samples were also repeated without the extensive initial preevaporation of layers, using different growth batches of caprolactam crystals, which are expected to have different spatial distributions of the filled $\mathrm{H}_{2} \mathrm{O}$ microinclusions. The $\sim 60 \AA$ wetting layer appeared in some samples but not in others. In one sample, which was taken from a 3-year old batch from the original experiments and was therefore expected to have extensive postgrowth exposure to $\mathrm{H}_{2} \mathrm{O}$, this transition in the wetting layer thickness to $\sim 60 \AA$ can be observed $12 \mathrm{~K}$ below the initial prewetting temperature reported in Ref. 4. Furthermore, the behavior of the wetting layer at higher temperatures was erratic and sample dependent. Clearly, this cannot be due to a thermodynamic process such as surface melting. This observed behavior, however, can be understood within the new proposed framework. Since different distributions or concentrations of microinclusions can influence the kinetics of the excavation process, it is not surprising that the wetting layer can appear at dramatically different temperatures.

It is unlikely, however, that the observed wetting layer is simply $\mathrm{H}_{2} \mathrm{O}$. Water has a higher electron density than solid caprolactam, whereas the layer wetting the surface is observed to have a $\sim 12 \%$ lower density instead, both in Ref. 4 and in this study. We propose that the layer that wets the solid surface is hydrated caprolactam. This provides a possible explanation for the deviation of the layer electron density from the bulk liquid value, which is $7 \%$ lower than the solid density instead of the observed $12 \%$. Moreover, the reported layer spacing implied by the stepwise thickness transitions is $\sim 10 \%$ longer than the hydrogen bonded bimolecular unit of caprolactam [Fig. 2(a)], but corresponds roughly to the length of the hydrated unit, in which such hydrogen bonds are replaced by bridging $\mathrm{H}_{2} \mathrm{O}$ molecules [Fig. 2(b)]. This interpretation also provides a possible explanation for the well-defined thickness of the wetting layer at $\sim 60 \AA$. For the situation of complete wetting of a finitevolume fluid on a solid substrate (as in the case for the ex- 


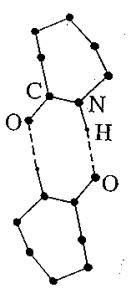

(a)

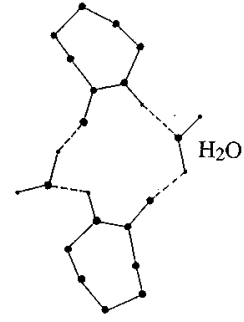

(b)
FIG. 2. (a) A representation of a bimolecular dimer unit of caprolactam: Only the $\mathrm{H}$ atoms in the hydrogen bonds between $\mathrm{N}$ and $\mathrm{O}$ atoms of the monomers are shown. The unmarked atoms in the top monomer are all $\mathrm{C}$ atoms. (b) The same unit after hydration: The hydrogen bonds now incorporate bridging $\mathrm{H}_{2} \mathrm{O}$ molecules.

cavation of filled inclusions), the spreading parameter $b$ will tend to thin the wetting layer in order to produce complete coverage of the solid surface. However, if the wetting layer has a lower electron density than the solid substrate, as in the present case, then the Hamaker constant $W$ is positive, ${ }^{8}$ and the van der Waals interactions tend to thicken the wetting layer. Due to this competition between surface interactions and van der Waals forces, the resultant film profile will not be a monolayer, but a wetting "pancake" with a welldefined thickness of $\sim(W / b)^{1 / 2} \cdot{ }^{9}$ For the present experiment, these constants correspond to those of the hydrated system, with a vapor-hydrated caprolactam interface and a hydrated caprolactam-solid caprolactam interface. This thickness of the wetting layer determined by the length scale $(W / b)^{1 / 2}$ is usually comparable to molecular dimensions, so the observed thickness of $\sim 60 \AA$ in the present context is not unreasonable.

In summary, we have repeated and reproduced the measurements of Chandarvarkar et al. using several variations of the original experiment, and find that the wetting behavior reported for caprolactam is inconsistent with surface melting via a prewetting transition. Further, we propose a surface hydration mechanism that can account for results from all the extant experiments thus far, as well as explain the origins of several empirical observations in the original experiment, which were not satisfactorily addressed by the surface melting hypothesis.

We acknowledge many helpful discussions with J. F. van der Veen, J. W. M. Frenken, and S. Chandavarkar. This work is part of the research program of the Stichting voor Fundamenteel Onderzoek der Materie [Foundation for Fundamental Research on Matter (FOM)] and was made possible by financial support from the Nederlandse Organisatie voor Wetenschappelijk Onderzoek [Netherlands Organization for the Advancement of Research (NWO)].
${ }^{1}$ For recent reviews, see, for example, (a) J. W. M. Frenken and H. M. van Pinxteren, in The Chemical Physics of Solid Surfaces, edited by D. A. King and D. P. Woodruff (Elsevier, Amsterdam, 1994), Vol. 7; (b) J. F. van der Veen, in Phase Transitions in Surface Films 2, edited by H. Taub et al. (Plenum, New York, 1991); (c) H. Dosch, in Critical Phenomena at Surfaces and Interfaces, Springer Tracts in Modern Physics Vol. 126 (Springer, Berlin, 1992).

${ }^{2}$ A. A. Chernov and V. A. Yakovlev, Pis'ma Zh. Eksp. Teor. Fiz. 45, 130 (1987) [JETP Lett. 45, 160 (1987)]; Langmuir 3, 635 (1987).

${ }^{3}$ W. H. de Jeu, J. D. Shindler, S. Chandavarkar, R. M. Geertman, and K. S. Liang, Surf. Sci. 342, 341 (1995).

${ }^{4}$ S. Chandavarkar, R. M. Geertman, and W. H. de Jeu, Phys. Rev. Lett. 69, 2384 (1992).

${ }^{5}$ R. M. Geertman and A. E. D. van der Heijden, J. Cryst. Growth 125, 303 (1992).
${ }^{6}$ J. Als-Nielsen, in Structure and Dynamics of Surfaces I, edited by W. Schommers and P. von Blackenhagen, Topics in Current Physics Vol. 41 (Springer-Verlag, Berlin, 1986); B. Pluis, J. M. Gay, J. W. M. Frenken, S. Gierlotka, J. F. van der Veen, J. E. Macdonald, A. A. Williams, N. Piggins, and J. Als-Nielsen, Surf. Sci. 222, L845 (1989).

${ }^{7}$ E. P. G. van den Berg, Ph.D. thesis, University of Nijmegen, 1996.

${ }^{8}$ We have assumed that the dielectric function does not have a complex frequency dependence. See, for example, M. Elbaum and M. Schick, Phys. Rev. Lett. 66, 1713 (1991).

${ }^{9}$ This can be obtained by dimensional analysis. For more details, see, for example, P. G. de Gennes, Rev. Mod. Phys. 57, 827 (1985); or S. A. Safran, Statistical Thermodynamics of Surfaces, Interfaces, and Membranes (Addison-Wesley, New York, 1994). 